\title{
Hilbert schemes of a surface and Euler characteristics
}

\author{
Mark Andrea A. de Cataldo*
}

September 22, 1998

\begin{abstract}
We use basic algebraic topology and Ellingsrud-Stromme results on the Betti numbers of punctual Hilbert schemes of surfaces to compute a generating function for the Euler characteristic numbers of the Douady spaces of "n-points" associated with a complex surface. The projective case was first proved by L. Göttsche.
\end{abstract}

\section{Introduction}

This note is dedicated to the determination of the Euler characteristic numbers $e\left(X^{[n]}\right)$ of the Douady spaces of "n-points" $X^{[n]}$ associated with a complex surface $X$; see Theorem 2.1.

For $X$ algebraic (the Douady space is replaced by the Hilbert scheme) see [9], [10] and [4]. In the projective case L. Göttsche [9] used P. Deligne's solution to the Weil Conjectures and EllingsrudStromme's determination of the Betti numbers of punctual Hilbert schemes [7]. Later, GöttscheSoergel [10] proved the quasi-projective case using the methods of perverse sheaves. J. Cheah [4] obtained similar results using the theory of Mixed Hodge structures and [7].

The proof presented here, valid without the algebraicity restriction on $X$, uses [ 7 and elementary algebraic topology.

Acknowledgments. I wish to the thank the Max-Planck-Institut für Mathematik in Bonn for its warm hospitality and stimulating environment. I would like to thank Pavlos Tzermias for useful conversations.

\section{Notation and preliminaries}

The topological Euler characteristic of a topological space $Y$, with respect to singular cohomology with compact supports, is denoted by $e(Y)$. If a statement involves this quantity $e(Y)$, it is understood that part of the statement consists of the assertion that $e(Y)$ is defined.

The term complex surface denotes, here, a smooth, connected and complex analytic surface with separable topology.

Let $X$ be a complex surface and $n \in \mathbb{N}^{*}$. Denote by $X^{[n]}$ the Douady Space [6] of zero-dimensional analytic subspaces of $X$ of length $n$; in the algebraic case this notion is replaced by the one of the Hilbert scheme; note that the "local differential geometries" of the Hilbert scheme and of the Douady spaces run parallel so that one can translate most of the proofs in $[8]$ in the analytic context. The spaces $X^{[n]}$ are connected, complex manifolds of dimension $2 n$. The corresponding Barlet space (cf. [1]) of zero-dimensional cycles in $X$ of total multiplicity $n$ can be identified with the symmetric product $X^{(n)}=X^{n} / \Sigma_{n}$, where $\Sigma_{n}$ denotes the symmetric group over $n$ elements and it acts by permuting the

\footnotetext{
${ }^{*}$ Partially supported by N.S.F. Grant DMS 9701779 and by an A.M.S. Centennial Fellowship.
} 
factors of $X^{n}$. There is a natural proper holomorphic map $\pi: X^{[n]} \rightarrow X^{(n)}$ which is a resolution of the singularities of $X^{(n)}$ (cf. [1] ); we call this morphism the Douady-Barlet morphism.

There is a natural stratification for this morphism given by the partitions of $n$. Let $P(n)$ denote the set of partitions of the natural number $n$ :

$$
P(n):=\left\{\nu=\left(\nu_{1}, \ldots, \nu_{k}\right) \mid k \in \mathbb{N}^{*}, \nu_{j} \in \mathbb{N}^{*}, \nu_{1} \geq \nu_{2} \geq \ldots \geq \nu_{k}>0, \sum_{j=1}^{k} \nu_{j}=n\right\} .
$$

The cardinality of $P(n)$ is denoted by $p(n)$. Every partition $\nu=\left(\nu_{1}, \ldots, \nu_{k}\right)$ can also be represented by a sequence of $n$ nonnegative integers:

$$
\nu=\left(\nu_{1}, \ldots, \nu_{k}\right) \Longleftrightarrow\left\{\alpha_{1}, \ldots, \alpha_{n}\right\},
$$

where $\alpha_{i}$ is the number of times that the integer $i$ appears in the sequence $\left(\nu_{1}, \ldots, \nu_{k}\right)$. Clearly $n=\sum_{i=1}^{n} i \alpha_{i}$. The number $k$ associated with $\nu$ is called the length of $\nu$ and it is usually denoted by $\lambda(\nu)$. The set $P(n)$ is in natural one to one correspondence with a set of mutually disjoint subsets of $X^{(n)}$ which are locally closed for the Zariski topology on analytic spaces: associate with every $\nu \in P(n)$ the space

$$
S_{\nu}^{n} X=S_{\left(\nu_{1}, \ldots, \nu_{k}\right)}^{n} X:=\left\{\sum_{j=1}^{k} \nu_{j} x_{j} \in X^{(n)} \mid x_{j_{1}} \neq x_{j_{2}} \text { if } j_{1} \neq j_{2}\right\} .
$$

The space $X^{(n)}$ is the disjoint union of these locally closed subsets:

$$
X^{(n)}=\coprod_{\nu \in P(n)} S_{\nu}^{n} X
$$

Define $\widetilde{S_{\nu}^{n} X}:=\pi^{-1}\left(S_{\nu}^{n} X\right)$. The space $X^{[n]}$ is the disjoint union of these locally closed subsets:

$$
X^{[n]}=\coprod_{\nu \in P(n)} \widetilde{S_{\nu}^{n} X} .
$$

Given $m \in \mathbb{N}^{*}$, the analytic isomorphism class of the fiber over a point $x \in X=S_{(m)}^{m} X$ of the Douady-Barlet morphism $\pi: X^{[m]} \rightarrow X^{(m)}$ are independent of the surface $X$ and of the point $x \in X$; since they are isomorphic to the fiber in question when $X$ is the affine plane $\mathbb{A}^{2}=\mathbb{C}^{2}$ and the point $x$ is the origin, we denote them by $\mathbb{A}_{[0]}^{2^{[m]}}$.

Theorem 1.1 (Cf. []) $e\left(\mathbb{A}_{[0]}^{2^{[m]}}\right)=p(m)$.

Results of Fogarty's [8] (see also [9]), adapted in this analytic context, ensure that the restriction of $\pi, \pi: \widetilde{S_{\nu}^{n} X} \rightarrow S_{\nu}^{n} X$, is a locally trivial fiber bundle in the analytic Zariski topology with fiber $F_{\nu}$

$$
F_{\nu} \simeq \prod_{j=1}^{k} \mathbb{A}_{[0]}^{2^{\left[\nu_{j}\right]}}
$$

We shall deal only with complex analytic spaces $Z$ for which the singular cohomology with compact supports satisifes $\operatorname{dim}_{\mathbb{Q}} H_{c}^{*}(W, \mathbb{Q})<\infty$; in particular the Euler numbers $e(Z)$ are defined. AlexanderSpanier cohomology, singular cohomology and sheaf cohomology with constant coefficients (all with compact supports) are naturally isomorphic to each other on the spaces that we will consider.

Alexander-Spanier's Theory has good properties with respect to closed subsets. We shall need two of these properties. In what follows we assume that $W, Y, Z$ and $Z_{i}$ are analytic spaces with finite 
dimensional rational cohomology with compact supports. Let $Z=\coprod_{i=1}^{n} Z_{i}$ where $\left\{Z_{i}\right\}$ is a finite collection of mutually disjoint locally closed subsets of $Z$. Then

$$
e(Z)=\sum_{i=1}^{n} e\left(Z_{i}\right)
$$

Let $f: W \rightarrow Z$ be a continuous map which is locally trivial, with fiber $Y$, with respect to the stratification $\Sigma_{i}$, i.e. $f^{-1}\left(Z_{i}\right) \simeq \Sigma_{i} \times Y$ for every $i=1, \ldots, n$. Then

$$
e(W)=e(Y) e(Z) .
$$

It should be now clear what we should be aiming at: reduce the computation of $e\left(X^{[n]}\right)$ to the

computation of $e\left(\widetilde{S_{\nu}^{n} X}\right)$ via (4). The numbers $e\left(\widetilde{S_{\nu}^{n} X}\right)$ will be computed using (5) and (3). The resulting sum will turn out to be a multinomial expansion.

\section{Statement of the result and the numbers $e\left(S_{\nu}^{(n)} X\right)$}

The following is the main result of this note.

Theorem 2.1 Let $X$ be a smooth complex surface such that $\operatorname{dim}_{\mathbb{Q}} H_{c}^{*}(X, \mathbb{Q})<\infty$ and with topological Euler characteristic $e(X)$. Let $X^{[n]}$ be the associated Douady spaces of " $n$-points." The following is a generating function for $e\left(X^{[n]}\right)$ :

$$
\sum_{n=0}^{\infty} e\left(X^{[n]}\right) q^{n}=\prod_{k=1}^{\infty}\left(\frac{1}{1-q^{k}}\right)^{e(X)} .
$$

Remark 2.2 The assumptions of the theorem are satisfied for any algebraic surface.

The proof will be preceded by some elementary calculations.

The structure of $S_{\nu}^{n} X$. Let $\nu=(1, \ldots, 1) \in P(n)$. The stratum $S_{(1, \ldots, 1)}^{n} X$ is the unique Zariski open subset in $X^{(n)}$ belonging to the stratification (11). Let $D^{n}$ be the big diagonal in the cartesian product $X^{n}$. The stratum $S_{(1, \ldots, 1)}^{n} X$ is the quotient of $X^{n} \backslash D^{n}$ under the natural free action of the symmetric group over $n$ elements $\Sigma_{n}$. It follows that $S_{(1, \ldots, 1)}^{n} X$ is smooth of complex dimension $2 n$. Each stratum $S_{\nu}^{n} X, \nu \in P(n)$, is built from these basic strata $S_{(1, \ldots, 1)}^{m} X$ for $m \leq n$. Here is how. Let $\nu=\left(\nu_{1}, \ldots, \nu_{k}\right) \leftrightarrow\left\{\alpha_{1}, \ldots, \alpha_{n}\right\}$ be the same partition in the two different pieces of notation. There is a natural isomorphism:

$$
S_{\nu}^{n} X \simeq\left(\prod_{i=1, \alpha_{i} \neq 0}^{n}\left(S_{(1, \ldots, 1)}^{\alpha_{i}} X\right)\right) \backslash \Delta
$$

where $\Delta$ is the closed set

$$
\Delta:=\left\{\left(C_{1}, \ldots, C_{l}\right) \in \prod_{i=1, \alpha_{i} \neq 0}^{n} S_{(1, \ldots, 1)}^{\alpha_{i}} X \mid \operatorname{Supp}\left(C_{h}\right) \cap \operatorname{Supp}\left(C_{j}\right) \neq \emptyset \text { for some } h \neq j\right\} .
$$

It follows that each stratum $S_{\nu}^{n} X$ is smooth, connected and of complex dimension $2 k=2 \lambda(\nu)$.

The numbers $e\left(S_{\nu}^{n} X\right)$. Recalling the convention $0 !=1$, we have the following elementary 
Lemma 2.3 Let $X$ be a smooth complex analytic surface with $\operatorname{dim}_{\mathbb{Q}} H^{*}(X, \mathbb{Q})<\infty, e=e(X), n \in \mathbb{N}^{*}$ and $P(n) \ni \nu=\left(\nu_{1}, \ldots, \nu_{k}\right) \leftrightarrow\left\{\alpha_{1}, \ldots, \alpha_{n}\right\}$. Then

$$
e\left(S_{\nu}^{n} X\right)=\frac{1}{\alpha_{1} ! \ldots \alpha_{n} !} e(e-1) \ldots(e-(\lambda(\nu)-1)) .
$$

Proof. We first compute $e\left(S_{(1, \ldots, 1)}^{m} X\right)$ for every $m$. Since $S_{(1, \ldots, 1)}^{m} X=\left(X^{m} \backslash D^{m}\right) / \Sigma_{m}$, and the action is free, we have that, by the multiplicativity of the number $e$ under finite covering maps, $m ! e\left(S_{1, \ldots, 1}^{m} X\right)=e\left(X^{m} \backslash D^{m}\right)$.

We now prove that $e\left(X^{m} \backslash D^{m}\right)=e(e-1) \ldots(e-(m-1)$ by induction on $m$. The case $m=1$ is trivial. Assume that the above formula is true for $m-1$ and let us prove it for $m$. We have a commutative diagram

$$
\begin{aligned}
X^{m} & \backslash D^{m} & \hookrightarrow & \left(X^{m-1} \backslash D^{m-1}\right) \times X \\
& \downarrow \operatorname{proj}_{1, \ldots, m-1} & & \swarrow \operatorname{proj}_{1} \\
X^{m-1} & \backslash D^{m-1} & &
\end{aligned}
$$

where the horizontal map is the natural open embedding, the vertical map is the projection to the first $(m-1)$ coordinates and the "south-west" map is the projection onto the first factor. The map $\operatorname{proj}_{1}$ admits $(m-1)$ natural sections $\left(x_{1}, \ldots, x_{m-1}\right) \rightarrow\left(x_{1}, \ldots, x_{m-1}, x_{l}\right)$, where $l=1, \ldots, m-1$. The images of these sections are pairwise disjoint. Their union is a closed subset of $\left(X^{m-1} \backslash D^{m-1}\right) \times X$ isomorphic to $(m-1)$ disjoint copies of $X^{m-1} \backslash D^{m-1}$; the complement of this set is the open subset which we identify with $X^{m} \backslash D^{m}$ via the open immersion in the diagram above. The additive and multiplicative properties of the number $e$ and the inductive hypothesis give that

$$
\begin{aligned}
e\left(X^{m} \backslash D^{m}\right) & =[e(e-1) \ldots(e-(m-1-1)) e]-[e(e-1) \ldots(e-(m-1-1))(m-1)] \\
& =e(e-1) \ldots(e-(m-1)) .
\end{aligned}
$$

This proves that

$$
m ! e\left(S_{1, \ldots, 1}^{m} X\right)=e\left(X^{m} \backslash D^{m}\right)=e(e-1) \ldots(e-(m-1)) .
$$

As to the case of $S_{\nu}^{n} X$ we note that, because of the isomorphism given in (7), this space is the quotient of $X^{\alpha_{1}+\ldots+\alpha_{n}=\lambda(\nu)} \backslash D^{\lambda(\nu)}$ under the free action of the group $\Sigma_{\alpha_{1}} \times \ldots \times \Sigma_{\alpha_{n}}$ induced by the individual actions of the groups $\Sigma_{\alpha_{i}}$ on the factors $X^{\alpha_{i}}$. The formula follows from the multiplicativity of Euler characteristics under finite covering maps.

Remark 2.4 It is an amusing exercise to derive Macdonald formula for the Euler characteristics of symmetric products using Lemma 2.3 and the multinomial expansion; i.e. prove that

$$
\sum_{n=0}^{\infty} e\left(X^{(n)}\right) q^{n}=\left(\frac{1}{1-q}\right)^{e(X)} .
$$

The numbers $e\left(\widetilde{S_{\nu}^{n} X}\right)$. By virtue of Lemma 2.3, Theorem 1.1 and (3), the basic additive and multiplicative properties of cohomology with compact support give the following

Lemma 2.5 Let $X$ be a complex surface with finite Betti numbers. Then

$$
\begin{aligned}
e\left(\widetilde{S_{\nu}^{n} X}\right) & =e\left(\prod_{j=1}^{\lambda(\nu)} \mathbb{A}_{[0]}^{2^{\left[\nu_{j}\right]}}\right) e\left(S_{\nu}^{n} X\right)=p\left(\nu_{1}\right) \ldots p\left(\nu_{k}\right) \frac{1}{\alpha_{1} ! \ldots \alpha_{n} !} e(e-1) \ldots(e-(\lambda(\nu)-1)) \\
& =p(1)^{\alpha_{1}} \ldots p(n)^{\alpha_{n}} \frac{1}{\alpha_{1} ! \ldots \alpha_{n} !} e(e-1) \ldots(e-(\lambda(\nu)-1)) .
\end{aligned}
$$




\section{Proof of Theorem 2.1}

Proof of Theorem 2.1. By virtue of (2), (1) and Lemma 2.5 we have

$$
\begin{aligned}
e\left(X^{[n]}\right) & =\sum_{\nu \in P(n)} e\left(\widetilde{S_{\nu}^{n} X}\right) \\
& =\sum_{k=1}^{n} \sum_{\substack{\nu \in P(n) \\
\lambda(\nu)=k}} \frac{p(1)^{\alpha_{1}} \ldots p(n)^{\alpha_{n}}}{\alpha_{1} ! \ldots \alpha_{n} !} e(e-1) \ldots(e-(k-1)) .
\end{aligned}
$$

The last summand is the expansion of the r.h.s. of equation (6). Let us check this for $e \geq 0$ from a combinatorial point of view. Set $p(0):=1$. For $e \geq 0$ the product $\prod_{k=1}^{\infty}\left(\frac{1}{1-q^{k}}\right)^{e(X)}$ can be defined as the limit, in $\mathbb{Q}[[q]]$, of the polynomials $P_{n}(q):=\left(\sum_{s=0}^{n} p(s) q^{s}\right)^{e}$. The coefficients $c_{m, s}$ of $q^{s}$ in $P_{m}(q)$ are the same as the one of the infinite product for every $s \leq n$ and every $m \geq n$. For $n \leq e$ their form is given by the multinomial coefficient expansion in the following way:

$$
\begin{aligned}
c_{m, s}= & \sum_{\substack{\sum_{i=0}^{n} a_{i}=e \\
\sum_{i=0}^{n} i a_{i}=n}}\left(\begin{array}{c}
e \\
a_{0}, \ldots, a_{n}
\end{array}\right) p(0)^{a_{0}} \ldots p(n)^{a_{n}} \\
= & \sum_{k=1}^{n} \sum_{\substack{\sum_{j=1}^{n} a_{j}=e-k \\
\sum_{j=1}^{n} j a_{j}=n}}^{\left(\begin{array}{c}
e \\
k, a_{1}, \ldots, a_{n}
\end{array}\right) p(1)^{a_{1}} \ldots p(n)^{a_{n}}} \\
= & \sum_{k=1}^{n} \sum_{\sum_{j=1}^{n} a_{j}=e-k} \frac{p(1)^{\alpha_{1}} \ldots p(n)^{\alpha_{n}}}{\alpha_{1} ! \ldots \alpha_{n} !} e(e-1) \ldots(e-(k-1)), \\
\sum_{j=1}^{n} j a_{j}=n &
\end{aligned}
$$

which coincides with $e\left(X^{[n]}\right)$. The formula is still correct for $n>e$ (many summands are zero). The case $e=0$ is trivial. We leave the case $e<0$ to the reader.

Remark 3.1 The generating function of Theorem 2.1 exhibits modular behavior (cf. [9]).

Remark 3.2 If $X$ is not algebraic, then the approach in [प] cannot be used to prove Theorem 2.1. The paper 10] restricts itself to the algebraic context. However, that restriction is unnecessary. This is remarked in [5], whose main purpose is to prove the necessary decomposition theorem in the form of an explicit quasi-isomorphism of complexes.

Remark 3.3 For $X$ algebraic the numbers $e\left(X^{[n]}\right)$ and the orbifold Euler characteristics $e\left(X^{n}, \Sigma_{n}\right)$ coincide. Similarly, we see that because of Theorem 2.1 this "coincidence" occurs for any complex surface, i.e. not necessarily algebraic. This is explained by the fact that the groups $K\left(X^{[n]}\right) \otimes_{\mathbb{Z}} \mathbb{Q}$ and the $\Sigma_{n}$-Equivariant K-Theory of $X^{n}, K_{\Sigma_{n}}\left(X^{n}\right) \otimes_{\mathbb{Z}} \mathbb{Q}$, are naturally isomorphic. This fact is due to B. Bezrukavnikov and V. Ginzburg [2] and, independently, to L. Migliorini and myself [5].

Question 3.4 The numbers $e\left(X^{[n]}\right)$ are invariant in the class of complex analytic smooth surfaces homotopically equivalent to $X$. Is $X^{[n]}$ a topological invariant of $X$ ? To be precise, let $X$ and $Y$ be complex surfaces homeomorphic to each other; is $X^{[n]}$ homeomorphic to $Y^{[n]}$ ? Is $X^{[n]}$ an invariant for the differentiable structure of $X$ ? 


\section{References}

[1] D. Barlet, Espace analytique réduit des cycles analytiques complexes compacts d'un espace analytique complexe de dimension finie, Lecture Notes in Mathematics 482, SPringer, Berlin, 1975.

[2] R. Bezrukavnikov, V. Ginzburg, "Hilbert schemes and reductive groups," preprint.

[3] J. Briançon, "Description de $H i l b^{n} C\{x, y\}$," Invent. Math. 41 (1977), 45-89.

[4] J. Cheah, "The Hodge numbers of the Hilbert scheme of points of a smooth projective surface," J. Algebraic Geometry 5 (1996), 479-511.

[5] M.A. de Cataldo, L. Migliorini, "The Douady space of a complex surface," in preparation.

[6] A. Douady, "Le problème des modules pour les sous-espaces analytiques d'un espace analytique donné," Ann. Inst. Fourier 16-1 (1966), 1-95.

[7] G. Ellingsrud, S.A. Stromme, "On a cell decomposition of the Hilbert scheme of points in the plane," Invent. Math. 91 (1988) 365-370.

[8] J. Fogarty, "Algebraic families on an algebraic surface," American Journal of Math. 90 (1968), 511-521.

[9] L. Göttsche, Hilbert schemes of zero-dimensional subschemes of smooth varieties, LNM 1572, Springer-Verlag Berlin 1994.

[10] L. Göttsche, W. Soergel, "Perverse sheaves and the cohomology of the Hilbert schemes of smooth algebraic surfaces," Math. Ann. 296 (1993), 235-245.

[11] F. Hirzebruch, H. Höfer, "On the Euler number of an orbifold," Math. Ann. 286, 255-260 (1990).

Author's address: Department of Mathematics, Harvard University, Science Center, One Oxford Street, Cambridge, MA 02138, U.S.A. e-mail: mde@math.harvard.edu 